IJMMS 25:10 (2001) 679-688

PII. S0161171201002794

http://ijmms.hindawi.com

(C) Hindawi Publishing Corp.

\title{
TAUTNESS AND APPLICATIONS OF THE ALEXANDER-SPANIER COHOMOLOGY OF $K$-TYPES
}

\author{
ABD EL-SATTAR A. DABBOUR and ROLA A. HIJAZI
}

(Received 5 February 1999)

\begin{abstract}
The aim of the present work is centered around the tautness property for the two $K$-types of Alexander-Spanier cohomology given by the authors. A version of the continuity property is proved, and some applications are given.
\end{abstract}

2000 Mathematics Subject Classification. Primary 55N05, 55N20, 55N35.

1. Introduction. It is well known that in the Alexander-Spanier cohomology theory $[17,18]$ or in the isomorphic theory of Čech [9], if the coefficient group $G$ is topological then either the theory does not take into account the topology on $G$ [9, 18], or considers only the case when $G$ is compact to obtain a compact cohomology [4, 1]. Continuous cohomology naturally arises when the coefficient group of a cohomology theory is topological [2, 3, 11]. The partially continuous Alexander-Spanier cohomology theory [14] can be considered as a variant of the continuous cohomology of a space with two topologies in the sense of Bott-Haefliger [15]; also it is isomorphic to the continuous cohomology of a simplicial space defined by Brown-Szczarba [2].

The idea of $K$-groups [5, 6], where $K$ is a locally-finite simplicial complex, is used to introduce the $K$-types of Alexander-Spanier cohomology with coefficients in a pair $\left(G, G^{\prime}\right)$ of topological abelian groups [7, 8]; namely, $K$-Alexander-Spanier and partially continuous $K$-Alexander-Spanier cohomologies $\bar{H}_{K}^{*}, \tilde{H}_{K}^{*}$. It is proved that these $K$-types satisfied the seven Eilenberg-Steenrod axioms [9]; the excision axiom for the second $K$-type is verified for compact Hausdorff spaces when $\left(G, G^{\prime}\right)$ are absolutely retract. Therefore, the uniqueness theorem of the cohomology theory on the category of compact polyhedral pairs [9] asserts that our Alexander-Spanier $K$-types over a pair of absolute retract coefficient abelian groups are naturally isomorphic.

In the present work, we prove that the $K$-Alexander-Spanier cohomology of a closed subset in a paracompact space is isomorphic to the direct limit of the $K$-AlexanderSpanier cohomology of its neighborhoods, and that the partially continuous $K$ Alexander-Spanier cohomology of a neighborhood retract closed subspace of a Hausdorff space is isomorphic to the direct limit of the partially continuous $K$-AlexanderSpanier cohomology of its neighborhoods. Also a version of the continuity property is proved. Moreover, we study some applications of the $K$-type cohomologies.

2. Alexander-Spanier cohomology of $K$-types. Here we mention the notations which we used throughout $[7,8]$.

For an object $(X, A)$ of the category $Q$ of the pairs of topological spaces and their continuous maps, denote by $\Omega(X, A)[\tilde{\Omega}(X, A)]$ the set of the pairs $\bar{\alpha}=\left(\alpha, \alpha^{\prime}\right)$, where 
$\alpha$ is an open covering of $X$ and $\alpha^{\prime}$ is a subcollection of $\alpha$ covering $A\left[\alpha^{\prime}=\alpha \cap A\right]$; it is directed with respect to the refinement relation $\bar{\alpha}<\bar{\beta}$, that is, $\alpha<\beta$ and $\alpha^{\prime}<\beta^{\prime}$ [9]. Denote by $C^{q(\tau)}(\tilde{X})$ the group of functions $\varphi^{\tau}: \tilde{X}^{q(\tau)+1} \rightarrow G$, where $\tau$ is a simplex in $K, q(\tau)=q+\operatorname{dim} \tau, q \geq 0$, and $\tilde{X}$ denotes either a space $X$ or $\alpha \in \Omega(X)$. Let $C^{q(\tau)}(\tilde{X})$ be the subgroup of the direct product $\prod_{\tau \in K} C^{q(\tau)}(\tilde{X})$ consisting of such $\varphi=\left\{\varphi^{\tau}\right\}$ for which the condition $(k)$ is satisfied, which states that there is a cofinite subset $\breve{\tau}(\varphi)$ of $K$, that is, $K-\breve{\tau}(\varphi)$ is finite such that $\left(\varphi^{\tau}\right)^{-1}\left(G^{\prime}\right)=\tilde{X}^{q(\tau)+1}, \forall \tau \in \breve{T}(\varphi)$. The coboundary $\delta^{q}: C^{q}(\tilde{X}) \rightarrow C^{q+1}(\tilde{X})$ is given by

$$
\left(\delta^{q} \varphi\right)^{\tau}=\sum_{i=1}^{q(\tau)+1}(-1)^{i} \varphi^{\tau} p_{i}^{(q(\tau)+1)}+(-1)^{q(\tau)+1} \sum_{\sigma \in \operatorname{st}(\tau)}[\sigma: \tau] \varphi^{\sigma},
$$

where $\operatorname{st}(\tau)=\{\sigma \in K: \tau$ is $(\operatorname{dim} \sigma-1)$-face of $\sigma\}, p_{i}^{(\tau)}: X^{\tau+1} \rightarrow X^{\tau}$ is the projection defined by: if $\hat{t}_{i}$ is the $\tau$-tuple consisting of $t=\left(x_{0}, \ldots, x_{\tau}\right) \in X^{\tau+1}$ with $x_{i}$ omitted, then $p_{1}^{(\tau)}(t)=\hat{t}_{i}, 0 \leq i \leq \tau$. The cohomology groups of the cochain complex $C^{\neq}(X)=$ $\left\{C^{q}(X), \delta^{q}\right\}$ is, in general, uninteresting, as shown in the following theorem [8].

THEOREM 2.1. If $\operatorname{dim} K=0$, then $H^{q}\left(C^{\neq}(X)\right) \cong G^{* K}$ (the subgroup of $G^{K}=\prod_{\tau \in K} G^{\tau}$, $G^{\top}=G$, consisting of those elements having all but a finite number of their $\tau$-coordinates in $\left.G^{\prime}\right)$, and $H^{q}\left(C^{\neq}(X)\right)=0$, when $q \neq 0$.

To pass to more interesting cohomology groups, the topology of the space $X$ will be used to define that $\varphi \in C^{q}(X)$ is said to be $K$-locally zero on $M \subseteq X$ if there is $\alpha \in \Omega_{X}(M)$ (the set of external covering of $M$ by open subsets of $X$ ) such that $\varphi$ vanishes on $\alpha \cap M$, that is, each $\varphi^{\tau}$ vanishes on $(\alpha \cap M)^{q(\tau)+1}$, where $\alpha^{\tau}=\cup\left\{u_{\alpha}^{\tau}\right.$ : $\left.u_{\alpha} \in \alpha\right\}$. The subgroups of $C^{q}(X)$ consisting of those elements which are $K$-locally zero on $X, A$, respectively, are denoted by $C_{0}^{q}(X), C^{q}(X, A)$. The $K$-Alexander-Spanier cohomology of $(X, A)$ over $\left(G, G^{\prime}\right)$, denoted by $\bar{H}_{K}^{*}(X, A)$, is the cohomology of the quotient cochain complex $\bar{C}_{K}^{\neq}(X, A)=C^{\neq}(X, A) / C_{0}^{\neq}(X)$. If $f:(X, A) \rightarrow(Y, B)$ is in $Q$, $\bar{\beta} \in \Omega(Y, B)$ and $\bar{\alpha}=f^{-1}(\bar{\beta})$, then $f$ defines a cochain map $\bar{f}^{\neq}: \bar{C}_{K}^{\neq}(Y, B) \rightarrow \bar{C}_{K}^{\neq}(X, A)$, where $\breve{T}\left(f^{q} \varphi\right)=\breve{T}(\varphi)$ for each $\varphi \in C^{q}(Y)$. In turn, $\bar{f}^{\neq}$induces the homomorphism $\bar{f}^{*}: \bar{H}_{K}^{*}(Y, B) \rightarrow \bar{H}_{K}^{*}(X, A)$.

On the other hand, for $\bar{\alpha} \in \Omega(X, A)$, denote by $C_{\bar{\alpha}}^{q}$ the subgroup of $C_{\alpha}^{q}=C^{q}(\alpha)$ consisting of those $\varphi$ that vanish on $\alpha^{\prime} \cap A$. Then we obtain a direct system $\left\{C_{\bar{\alpha}}^{ \pm}\right\}_{\Omega(X, A)}$ such that any map $f \in Q$ constitutes a map $F:\left\{C_{\bar{\beta}}^{\neq}\right\}_{\Omega(Y, B)} \rightarrow\left\{C_{\bar{\alpha}}^{\neq}\right\}_{\Omega(X, A)}$ [9]; its limit is $F^{\infty}$.

THEOREM 2.2. The K-Alexander-Spanier cohomology functor $\left\{\bar{H}_{K}^{*}, \bar{f}^{*}\right\}$ is naturally isomorphic to the functor $\left\{\lim _{\longrightarrow}\left\{H^{*}\left(C_{\bar{\alpha}}^{ \pm}\right)\right\}_{\Omega(X, A)}, F^{\infty *}\right\}$ [7].

In the previous part, the topology on $(G, G)$ plays no role; to pass to the second cohomology of $K$-type we characterize an element $\varphi \in C^{q}(X)$ to be $K$-partially continuous if it is continuous on some $\alpha \in \Omega(X)$, that is, $\varphi^{\tau} \mid \alpha^{q(\tau)+1}$ are continuous functions. Let $L^{q}(X)$ be the group of all such elements, and $M_{K}^{\neq}(X)=L^{\neq}(X) / C_{0}^{\neq}(X)$. The subgroup of $C_{\alpha}^{q}$, where $\alpha \in \Omega(X)$, consisting of the $K$-continuous elements $\varphi$, that is, $\varphi^{T}$ are continuous, is denoted by $M_{\alpha}^{q}$. Let $i: A \hookrightarrow X$, define $M_{K}^{ \pm}(X, A)$ to be the mapping cone of $i^{\neq}: M_{K}^{\neq}(X) \rightarrow M_{K}^{\neq}(A)$, (see $[13,18]$ ), assuming that $M_{K}^{q}(X, A)=M_{K}^{q}(X) \oplus M_{K}^{q-1}(A)$, and 
the coboundary is $\triangle^{q}(\varphi, \psi)=\left(-\delta^{q} \varphi, i^{q} \varphi+\delta^{q-1} \psi\right)$. The cohomology of $M_{K}^{ \pm}(X, A)$ is the partially continuous $K$-Alexander-Spanier cohomology of $(X, A)$ over the topological pair $\left(G, G^{\prime}\right)$ of coefficient groups; it is denoted by $\tilde{H}_{K}^{*}(X, A)$.

On the other hand, if $\bar{\alpha} \in \tilde{\Omega}(X, A)$, then $i$ defines a cochain map $i_{\alpha}^{\neq}: M_{\alpha}^{\neq} \rightarrow M_{\alpha^{\prime}}^{\neq}$; its mapping cone is denoted by $M_{\bar{\alpha}}^{\neq}$.

THEOREM 2.3. For a pair $(X, A) \in Q$ with $A$ closed, $M_{K}^{ \pm}(X, A)$ is naturally isomorphic to $\lim _{\longrightarrow}\left\{M_{\bar{\alpha}}^{ \pm}\right\}_{\tilde{\Omega}(X, A)}[7]$.

THEOREM 2.4. For a discrete space, and $q \geq 0, \tilde{H}_{K}^{q}(X) \cong \bar{H}_{K}^{q}(X)$.

Proof. Since $X^{q(\tau)+1}$ admits a discrete topology, it follows that each $\tau$-coordinate $\varphi^{\top}$ of $\varphi \in C_{K}^{q}(X)$ is continuous [16]. Then $\varphi$ is $K$-partially continuous with respect to any $\alpha \in \Omega(X)$. Therefore, $L^{q}(X)=C_{K}^{q}(X)$ and $M_{K}^{\neq}(X)=\bar{C}_{K}^{ \pm}(X)$.

3. Tautness and continuity properties. This article is devoted to study the tautness property for both Alexander-Spanier cohomology of $K$-types. One of its applications is the continuity property.

The star of a subset $A$ in a space $X$ with respect to $\alpha \in \Omega(X)$ is

$$
\operatorname{st}(A, \alpha)=\cup\left\{U_{\alpha} \in \alpha: U_{d} \cap A \neq \varnothing\right\} .
$$

The star of $\alpha$ is

$$
\alpha^{*}=\left\{\operatorname{st}\left(U_{\alpha}, \alpha\right): u_{\alpha} \in \alpha\right\} .
$$

DeFinItion 3.1. Let $\alpha, \beta \in \Omega(X)$, then $\beta$ is a star-refinement of $\alpha$, written $\alpha<^{*} \beta$ if $\alpha<\beta^{*}$.

Denote by $\mathcal{N}(A)$ the collections of neighborhoods $\{N\}$ of $A$ in $X$; it is directed downward by inclusion. If $N_{1}<N_{2}$, then the inclusion $\pi_{N_{1} N_{2}}: N_{2} \hookrightarrow N_{1}$ induces the homomorphisms $\bar{\pi}_{N_{1} N_{2}}^{*}: \bar{H}_{K}^{q}\left(N_{1}\right) \rightarrow \bar{H}_{K}^{q}\left(N_{2}\right)$. Also $i_{N}: A \hookrightarrow N$ induces $\bar{i}_{N}^{*}: \bar{H}_{K}^{q}(N) \rightarrow \bar{H}_{K}^{q}(A)$, and they define a homomorphism

$$
I^{\infty}: \underline{\lim }\left\{\bar{H}_{K}^{q}(N), \bar{\pi}_{N_{1} N_{2}}^{*}\right\}_{\mathcal{N}(A)} \longrightarrow \bar{H}_{K}^{q}(A) .
$$

THEOREM 3.2 (Tautness). A closed subspace of a paracompact space is a taut subspace relative to the $K$-Alexander-Spanier cohomology, that is, $I^{\infty}$ is an isomorphism for each $q$ and any pair $\left(G, G^{\prime}\right)$ of coefficient groups.

Proof. (1) $I^{\infty}$ is an epimorphism. Let $h \in \bar{H}_{K}^{q}(A)$ with representative $\bar{\varphi} \in \bar{C}_{K}^{q}(A)$, written as $h=[\bar{\varphi}]$. Let $\varphi \in C^{q}(A)$ such that $\varphi \in \bar{\varphi}$. Then there is $\alpha=\left\{u_{\alpha}=v_{\alpha} \cap A\right.$ : $v_{\alpha} \subseteq X$ is open $\} \in \Omega(A)$ such that

$$
\left(\delta^{q} \varphi\right) \mid \alpha^{q(\tau)+2}=0 .
$$

Since $A$ is closed, it follows that $\beta=\left\{v_{\alpha}\right\} \cup\{X-A\} \in \Omega(X)$. The paracompactness of $X$ is equivalent to the existence of such $\gamma \in \Omega(X)$ that $\beta<^{*} \gamma$, and a neighborhood $N$ of $A$ and an extension $f: N \rightarrow A$ (not necessarily continuous) of the identity map id id $_{A}$ of $A$, that is, $f i_{N}=\mathrm{id}_{A}$, such that $f\left(u_{\gamma} \cap N\right) \subseteq \operatorname{st}\left(u_{\gamma}, \gamma\right)$ for each $u_{\gamma} \in \gamma$ [18]. One can show that $f$ defines a cochain map $f^{\neq}: C^{\neq}(A) \rightarrow C^{\neq}(N)$ by $\left(f^{q} \varphi\right)^{\tau}=\varphi^{\tau} f^{(q(\tau)+1)}$ with 
$\breve{\tau}\left(f^{q} \varphi\right)=\breve{\tau}(\varphi)$, where $f^{(\tau)}: N^{\tau} \rightarrow A^{\tau}$ given by $f\left(x_{0}, \ldots, x_{\tau-1}\right)=\left(f\left(x_{0}\right), \ldots, f\left(x_{\tau-1}\right)\right)$. The relation $\beta<\gamma^{*}$ yields that for each $u_{\gamma} \in \gamma$ there is $u_{\beta} \in \beta$ such that $f\left(u_{\gamma} \cap N\right) \subseteq$ st $\left(u_{\gamma}, \gamma\right) \subseteq u_{\beta}$. Because $f(N)=A$, then $f\left(u_{\gamma} \cap N\right) \subseteq u_{\beta} \cap A \subseteq u_{\alpha}$ for some $u_{\alpha} \in \alpha$. By using (3.4), we get $\left(\delta^{q} f^{q} \varphi\right)^{\tau} \mid(\gamma \cap N)^{q(\tau)+2}=0$, that is, $\delta^{q}\left(f^{q} \varphi\right) \in C_{0}^{q+1}(N)$. Then $f^{q} \varphi$ represents a cocycle $\overline{f^{q} \varphi} \in \bar{C}_{K}^{q}(N)$ which, in turn, defines $h_{N} \in \bar{H}_{K}^{q}(N)$, that is, $h_{N}=\left[\overline{f^{q} \varphi}\right]$. Let $t \in A^{q(\tau)+1}$, then

$$
\left(i_{N}^{q}\left(f^{q} \varphi\right)\right)^{\tau}(t)=\varphi^{\tau} f^{(q(\tau)+1)} i_{N}^{(q(\tau)+1)}(t)=\varphi^{\tau}(t),
$$

and therefore, $\bar{i}_{N}^{*} h_{N}=\left[\overline{\left(f i_{N}\right)^{q} \varphi}\right]=[\bar{\varphi}]=h$.

(2) $I^{\infty}$ is a monomorphism. Let $h_{1} \in \bar{H}_{K}^{q}\left(N_{1}\right), \bar{\varphi}_{1} \in \bar{C}_{K}^{q}\left(N_{1}\right)$ and $\varphi_{1} \in C^{q}\left(N_{1}\right)$ such that $\varphi_{1} \in \bar{\varphi}_{1}, \bar{\varphi}_{1} \in h_{1}$, and $\left[h_{1}\right] \in \operatorname{Ker} I^{\infty}$.

First, one can consider that the neighborhood $N_{1}$ of $A$ is a paracompact subset of $X$. For, if $N_{1}$ is not so, then there is a paracompact subset $M_{1}$ of $X$ such that $M_{1}<N_{1}$ (e.g., take $M_{1}=X$ ) [10]. The inclusion $\pi_{M_{1} N_{1}}$ induces an epimorphism $\bar{\pi}_{M_{1} N_{1}}^{*}$ [8], let $\bar{\pi}_{M_{1} N_{1}}^{q} \bar{\psi}_{1}=\bar{\varphi}_{1}$. Thus the cohomology class of $\bar{H}_{K}^{q}\left(M_{1}\right)$ represented by $\bar{\psi}_{1}$ is $\left[h_{1}\right]$, which shows that $N_{1}$ can be taken paracompact.

Now, $\bar{\varphi}_{1} \in \operatorname{Ker} \delta^{q}$, or equivalently, there is $\alpha=\left\{u_{\alpha}=v_{\alpha} \cap N_{1}: v_{\alpha} \subseteq X\right.$ is open $\} \in$ $\Omega\left(N_{1}\right)$ such that

$$
\left(\delta^{q} \varphi_{1}\right)^{\tau} \mid \alpha^{q(\tau)+2}=0 .
$$

On the other hand, the assumption $\bar{i}_{N_{1}}^{*} h_{1}=0$ asserts that there exists $\bar{\varphi} \in \bar{C}_{K}^{q-1}(A)$ such that $i_{N_{1}}^{q} \varphi_{1}-\delta^{q-1} \varphi \in C_{0}^{q}(A)$, where $\varphi \in \bar{\varphi}$. This means that there is $\beta=\left\{u_{\beta}=\right.$ $\omega_{\beta} \cap A: \omega_{\beta} \subseteq X$ is open $\} \in \Omega(A)$ such that

$$
\left(i_{N_{1}}^{q} \varphi_{1}\right)^{\tau}=\left(\delta^{q-1} \varphi\right)^{\tau} \quad \text { on } \beta^{q(\tau)+1} .
$$

Assume that $\beta_{1}=\left\{u_{\beta_{1}}=\omega_{\beta} \cap N_{1}\right\} \cup\left\{N_{1}-A\right\}$. The paracompactness of $N_{1}$ asserts the existence of $\gamma_{1}, \gamma_{2} \in \Omega\left(N_{1}\right)$ for which $\alpha<^{*} \gamma_{1}$ and $\beta_{1}<^{*} \gamma_{2}$. The directedness of $\Omega\left(N_{1}\right)$ implies that there is $\gamma \in \Omega\left(N_{1}\right)$ for which $\gamma_{1}, \gamma_{2}<\gamma$; and so for each $u_{\gamma} \in \gamma$ there are $u_{\gamma_{i}} \in \gamma_{i}, i=1,2$ and $u_{\alpha} \in \alpha, u_{\beta_{1}} \in \beta_{1}$ such that

$$
u_{\gamma} \subset u_{\gamma_{i}} \subseteq \operatorname{st}\left(u_{\gamma_{i}}, \gamma_{i}\right) \subseteq u_{\alpha} \cap u_{\beta_{1}} .
$$

Then

$$
\text { st }\left(u_{\gamma}, \gamma\right) \subseteq u_{\alpha} \cap u_{\beta_{1}},
$$

that is, $\alpha, \beta_{1}<^{*} \gamma$. According to [18, Lemma 6.6.1], there is a neighborhood $N_{2}$ of $N_{1}$ and $f: N_{2} \rightarrow A$ (not necessarily continuous) such that $f i_{N_{2}}=\operatorname{id}_{A}$, and $u_{\beta_{1}} \in \beta_{1}$ such that

$$
f\left(u_{\gamma} \cap N_{2}\right) \subseteq \operatorname{st}\left(u_{\gamma}, \gamma\right) \subseteq u_{\beta_{1}} \subseteq u_{\beta_{1}} \cap A=u_{\beta} .
$$

Then, by (3.7), we get

$$
\left(\delta^{q-1} f^{q-1} \varphi\right)^{\tau}=\left(f^{q} i_{N_{1}}^{q} \varphi_{1}\right) \quad \text { on }\left(\gamma \cap N_{2}\right)^{q(\tau)+1} .
$$

Define $D^{q}: C^{q+1}\left(N_{1}\right) \rightarrow C^{q}\left(N_{2}\right)$ by

$$
\text { if } t=\left(x_{0}, \ldots, x_{q(\tau)}\right) \in N_{2}^{q(\tau)+1} \text { and } \psi_{1} \in C^{q+1}\left(N_{1}\right)
$$


then

$$
\left(D^{q} \psi_{1}\right)^{\tau}(t)=\sum_{r=0}^{q(\tau)}(-1)^{\gamma} \psi_{1}^{\tau}\left(y_{0}, \ldots, y_{\tau}, z_{\tau}, \ldots, z_{q(\tau)}\right),
$$

where

$$
y_{j}=\pi_{N_{1} N_{2}}\left(x_{j}\right), \quad z_{j}=\left(i_{N_{1}} f\right)\left(x_{j}\right)=f\left(x_{j}\right),
$$

and $\breve{\tau}\left(D^{q} \psi_{1}\right)=\breve{\tau}\left(\psi_{1}\right)$. By a similar calculation as given in [7], we get

$$
\left(\delta^{q-1} D^{q-1} \varphi_{1}\right)^{\tau}=\left(f^{q} i_{N_{1}}^{q} \varphi_{1}\right)^{\tau}-\left(\pi_{N_{1} N_{2}}^{q} \varphi_{1}\right)^{\tau}-\left(D^{q} \delta^{q} \varphi_{1}\right)^{\tau} .
$$

By (3.9), (3.10) for each $u_{\gamma} \in \gamma$, there is $u_{\alpha} \in \alpha$ such that

$$
\left(u_{\gamma} \cap N_{2}\right) \cup f\left(u_{\gamma} \cap N_{2}\right) \subseteq u_{\alpha} .
$$

Then, by (3.6), (3.11), and (3.15) consequently, we have

$$
\left(\delta^{q-1} D^{q-1} \varphi_{1}\right)^{\tau}=\left(f^{q} i_{N_{1}}^{q} \varphi_{1}\right)^{\tau}-\left(\pi_{N_{1} N_{2}}^{q} \varphi_{1}\right)^{\tau} \quad \text { on }\left(\gamma \cap N_{2}\right)^{q(\tau)+1},
$$

and so

$$
\left(\pi_{N_{1} N_{2}}^{q} \varphi_{1}\right)^{\tau}=\left(\delta^{q-1}\left(f^{q-1} \varphi-D^{q-1} \varphi_{1}\right)\right)^{\tau} \quad \text { on }\left(\gamma \cap N_{2}\right)^{q(\tau)+1} .
$$

Therefore

$$
\psi_{2}=f^{q-1} \varphi-D^{q-1} \varphi_{1} \in C^{q-1}\left(N_{2}\right)
$$

such that

$$
\left(\pi_{N_{1} N_{2}}^{q} \varphi_{1}\right)^{\tau}=\left(\delta^{q-1} \psi_{2}\right)^{\tau} \quad \text { on }\left(\gamma \cap N_{2}\right)^{q(\tau)+1},
$$

that is, $\bar{\pi}_{N_{1} N_{2}} h_{1}=0$ which completes the proof.

COROLLARY 3.3. Any one-point subset of a paracompact is a taut subspace relative to $\bar{H}_{K}^{*}$.

The next part is devoted to studying the tautness property for $\tilde{H}_{K}^{*}$, which is also valid for $\bar{H}_{K}^{*}$. The idea and results of $\alpha$ - $\beta$-contiguous maps, introduced in [7] plays an essential role in this study.

The inclusions $\pi_{N_{1} N_{2}}: N_{2} \hookrightarrow N_{1}$, corresponding to the relations $N_{1}<N_{2}$ in $\mathcal{N}(A)$, define the direct system $\left\{\tilde{H}_{K}^{q}(N), \tilde{\pi}_{N_{1} N_{2}}^{*}\right\}$. Also the inclusion $i_{N}: A \hookrightarrow N$, where $N \in$ $\mathcal{N}(A)$, defines a map of direct systems [9]:

$$
I_{N}:\left\{H^{q}\left(M_{\alpha}^{\neq}\right), \tilde{\pi}_{\alpha \beta}^{*}\right\}_{\Omega(N)} \longrightarrow\left\{H^{q}\left(M_{\tilde{\alpha}}^{ \pm}\right), \tilde{\pi}_{\tilde{\alpha} \tilde{\beta}}^{*}\right\}_{\Omega(A)},
$$

where $\alpha \in \Omega(N), \tilde{\alpha}=i_{N}^{-1}(\alpha)=\alpha \cap A$. On the other hand, $\left\{\tilde{i}_{N}^{*}\right\}$ defines a homomorphism

$$
\tilde{I}^{\infty}: \underline{\lim }\left\{\tilde{H}_{K}^{q}(N), \tilde{\pi}_{N_{1} N_{2}}^{*}\right\}_{\mathcal{N}(A)} \longrightarrow \tilde{H}_{K}^{q} A
$$

THEOREM 3.4 (Tautness). If $A$ is a closed subset in a Hausdorff space $X$ such that $A$ is a neighborhood retract, then $A$ is a taut subspace relative to the cohomology $\tilde{H}_{K}^{*}$. 
Proof. (1) $\tilde{I}^{\infty}$ is an epimorphism. Let $h \in \tilde{H}_{K}^{q}(A)$, without loss of generality, the neighborhood retractness of $A$ in $X$ yields that $A$ has an open neighborhood $U$ (in $X$ ) such that $U \subseteq N$ and a retraction $\tau_{1}: U \rightarrow A$ (if $U_{1}$ is an open neighborhood of $A$ of which $A$ is retract but $U_{1} \nsubseteq N$, take $\left.U=U_{1} \cap \operatorname{Int} N\right)$. Let $i_{U}: A \hookrightarrow U$ then, $\tilde{I}^{\infty}\left[\tilde{\tau}_{1}^{*}(h)\right]=$ $\tilde{i}_{U}^{*}\left(\tilde{\tau}_{1}^{*} h\right)=\tilde{\mathrm{id}}_{A}^{*}(h)=h$.

(2) $\tilde{I}^{\infty}$ is a monomorphism. Let $[h] \in \operatorname{Ker} \tilde{I}^{\infty}$. It is sufficient to construct $V \in \mathcal{N}(A)$ satisfying $N<V$ and $\tilde{\pi}_{N V}^{*} h=0$. Since the cohomology functor commutes with the direct limit [18]. Theorem 2.3 asserts that one may assume that $h$ belongs to $\mathrm{lim}_{\longrightarrow}\left\{H^{q}\left(M_{\alpha}^{\neq}\right), \tilde{\pi}_{\alpha \beta}^{*}\right\}_{\Omega(N)}$ with representative $h_{\alpha} \in H^{q}\left(M_{\alpha}^{\neq}\right)$, where

$$
\alpha=\left\{u_{\alpha}=\omega_{\alpha} \cap N: \omega_{\alpha} \subseteq X \text { is open }\right\} \in \Omega(N) .
$$

Let $\alpha_{1}=\left\{\omega_{\alpha}\right\} \cup\{X-A\}, \tilde{\alpha}=\alpha_{1} \cap A$,

$$
\beta=\left\{u_{\beta}=\tau_{1}^{-1}\left(u_{\tilde{\alpha}}\right) \cap\left(u_{\alpha} \cap U\right): \phi \neq u_{\tilde{\alpha}} \in \tilde{\alpha}\right\}
$$

$V=\cup_{u \beta}, \tau=\tau_{1} \mid V: V \hookrightarrow A$, and $\alpha^{\prime}=\alpha_{1} \cap V$. Then $\tilde{\alpha} \in \Omega(A), \alpha^{\prime}=\alpha \cap V \in \Omega(V)$, $u_{\tilde{\alpha}} \subseteq u_{\beta}$ for each $u_{\tilde{\alpha}} \neq \phi, \beta$ is a family of open subsets in $U$ and so open in $X, V$ is an open neighborhood of $A$ such that $V \subseteq U$, and $\beta \in \Omega(V)$. Since $u_{\beta}=u_{\beta} \cap u_{\alpha} \subseteq$ $V \cap u_{\alpha}=u_{\alpha^{\prime}}$, it follows that $\alpha^{\prime}<\beta$. Also $\alpha^{\prime} \cap A=\alpha \cap A=\tilde{\alpha}$ and $j^{-1} \beta=\tilde{\alpha}$, where $j: A \hookrightarrow V$. If $\ell: V \hookrightarrow N$, and $[\varphi] \in H^{q}\left(M_{\alpha}^{\neq}\right)$, then

$$
\tilde{j}_{\beta}^{*} \tilde{\pi}_{\alpha^{\prime} \beta}^{*} \tilde{\ell}_{\alpha}^{*}[\varphi]=\tilde{j}_{\beta}^{*}\left[\left\{\left(\varphi^{\tau} \mid \alpha^{q(\tau)+1}\right) \mid \beta^{q(\tau)+1}\right\}\right]=\left[\left\{\varphi^{\tau} \mid \tilde{\alpha}^{q(\tau)+1}\right\}\right],
$$

that is,

$$
\tilde{j}_{\beta}^{*} \tilde{\pi}_{\alpha^{\prime} \beta}^{*} \tilde{\ell}_{\alpha}^{*}=\tilde{i}_{N, \alpha}^{*}
$$

where $\tilde{i}_{N, \alpha}^{ \pm}: M_{\alpha}^{\neq} \rightarrow M_{\tilde{\alpha}}^{\neq}$is induced by $i_{N}: A \hookrightarrow N$.

On the other hand, $(j \tau) u_{\beta} \subseteq u_{\beta}$ and so $j \tau, \mathrm{id}_{V}: V \rightarrow V$ are $\beta-\beta$-contiguous [7].

It follows that $\left(\tilde{\mathrm{id}}_{V}\right)_{\beta-\beta}^{q},(\widetilde{j r})_{\beta-\beta}^{q}: M_{\beta}^{q} \rightarrow M_{\beta}^{q}$ are cochain homotopic [7]. Then $\left(\tilde{\mathrm{id}}_{V}\right)_{\beta-\beta}^{*}$ $=(\widetilde{j r})_{\beta-\beta}^{*}=\tilde{r}_{\tilde{\alpha}-\beta}^{*} \tilde{j}_{\beta}^{*}$, which yields that $\tilde{j}_{\beta}^{*}$ is a monomorphism. Because $\tilde{i}_{N, \alpha}^{*} h_{\alpha}=0$, equation (3.26) yields that $\tilde{\pi}_{\alpha^{\prime} \beta}^{*} \tilde{\ell}_{\alpha}^{*} h_{\alpha}=0$. Since $\tilde{\ell}_{\alpha}^{*} h_{\alpha}, \tilde{\pi}_{\alpha^{\prime} \beta}^{*}\left(\tilde{\ell}_{\alpha} h_{\alpha}\right)$ represent the zero element of $\underline{\lim }_{\longrightarrow}\left\{H^{q}\left(M_{\alpha}^{\neq}\right), \tilde{\pi}_{\alpha \beta}^{*}\right\}_{\Omega(N)}$, it follows that $\tilde{\pi}_{N V}^{*} h=\left[\tilde{\ell}_{\alpha}^{*} h_{\alpha}\right]=0$.

The rest of this article is centered around a special case of the continuity property for $\bar{H}_{K}^{*}$. As an application of the continuity property the cohomology groups satisfy a much stronger form of the excision axiom.

The following results can be deduced from those given in [9].

LEMMA 3.5. Let $X$ be the intersection of a nested system $\left\{X_{\alpha}, \pi_{\beta \alpha}\right\}_{\Lambda}$, then

(i) $X$ and $\lim _{\{}\left\{X_{\alpha}, \pi_{\beta \alpha}\right\}_{\Lambda}$ are homeomorphic.

(ii) If the nested system consists of compact Hausdorff spaces then $X$ is a closed subset of each $X_{\alpha}$.

(iii) If $N$ is an open neighborhood of $X$ in $X_{\alpha}$ (for some $\alpha \in \Lambda$ ), then there is $\beta>\alpha$ in $\Lambda$ such that $X_{\beta} \subseteq N$. 
The inclusions $i_{\alpha}: X \hookrightarrow X_{\alpha}$ define a map

$$
I:\left\{\bar{H}_{K}^{q}\left(X_{\alpha}\right), \bar{\pi}_{\alpha \beta}^{*}\right\}_{\Lambda} \longrightarrow \bar{H}_{K}^{q}(X),
$$

its direct limit is denoted by $\bar{I}^{\infty}$.

THEOREM 3.6 (weak continuity). If $X$ is the intersection of a nested system $\left\{X_{\alpha}\right.$, $\left.\pi_{\beta \alpha}\right\}_{\Lambda}$ of compact Hausdorff spaces, then $\bar{I}^{\infty}$ is an isomorphism.

Proof. Since each $X_{\alpha}$ is a paracompact Hausdorff space [10] and $X_{\alpha}$ is closed in $X$ (Lemma 3.5), it follows, by Theorem 3.2, that $X$ is a taut subspace in $X_{\alpha}$ relative to $\bar{H}_{K}^{*}$.

(1) $\bar{I}^{\infty}$ is an epimorphism. Let $h \in \bar{H}_{K}^{q}(X)$, then, according to Theorem 3.2, there exists an open neighborhood $N$ of $X$ in $X_{\alpha}$ and $h_{N} \in \bar{H}_{K}^{q}(N)$, such that $\bar{i}_{N}^{*}\left(h_{N}\right)=h$. By Lemma 3.5, there is $\beta>\alpha$ in $\Lambda$ such that $X_{\beta} \subseteq N$. Let $i_{\beta}: X \hookrightarrow X_{\beta}, j_{\beta}: X_{\beta} \hookrightarrow N$. Because $\bar{i}_{\beta}^{*}\left(\bar{j}_{\beta}^{*} h_{N}\right)=\left(\overline{j_{\beta} i_{\beta}}\right)^{*} h_{N}=\bar{i}_{N}^{*} h_{N}=h$, then $\bar{I}^{\infty}\left[\bar{j}_{\beta}^{*} h_{N}\right]=h$.

(2) $\bar{I}^{\infty}$ is a monomorphism. Let $\left[h_{\alpha}\right] \in \operatorname{Ker} \bar{I}^{\infty}$, that is, $\bar{i}_{\alpha}^{*} h_{\alpha}=0$. The tautness of $X$ in $X_{\alpha}$ yields, by Theorem 3.2, an open neighborhood $N$ of $X$ in $X_{\alpha}$ such that $h_{N}$ is the unique element for which $\bar{i}_{N}^{\prime *} h_{N}=0$, where $i_{N}^{\prime}: X \hookrightarrow N$. Because $\bar{i}_{N}^{\prime *}\left(\bar{i}_{N}^{*} h_{\alpha}\right)=\bar{i}_{\alpha}^{*} h_{\alpha}=0$, then $\bar{i}_{N}^{*} h_{\alpha}=0$. Let $\beta>\alpha$ in $\Lambda$ such that $X_{\beta} \subseteq N$, then $\bar{\pi}_{\alpha \beta}^{*} h_{\alpha}=\left(\overline{i_{N} i_{\beta}}\right){ }^{*} h_{\alpha}=\bar{j}_{\beta}^{*}\left(\bar{i}_{N}^{*} h_{\alpha}\right)=$ 0 , that is, $\left[h_{\alpha}\right]=0$.

4. Applications. One of the good applications of the Alexander-Spanier cohomology of $K$-types is the study of the 0-dimensional cohomology groups and their relation with the connectedness of the space [7]. In this article, two applications are given. In a next work, we hope to give more applications. As a first application, we define the partially continuous $K$-Alexander-Spanier cohomology of an excision map and calculate its value for some dimensions.

Let $\tilde{f}^{\neq}: M_{K}^{\neq}(Y, B) \rightarrow M_{K}^{\neq}(X, A)$ be the cochain map induced by the map $f$ in $Q$. Define $M_{K}^{ \pm}(f)$ to be the mapping cone of $\tilde{f}^{\neq}$by

$$
\begin{aligned}
M_{K}^{q}(f) & =M_{K}^{q}(Y, B) \oplus M_{K}^{q-1}(X, A) \\
& =M_{K}^{q}(Y) \oplus M_{K}^{q-1}(B) \oplus M_{K}^{q-1}(X) \oplus M_{K}^{q-2}(A),
\end{aligned}
$$

and the coboundary is

$$
\begin{aligned}
\tilde{\triangle}^{q} & \left(\varphi_{2}, \psi_{2}, \varphi_{1}, \psi_{1}\right) \\
& =\left(-\tilde{\triangle}^{q}\left(\varphi_{2}, \psi_{2}\right), \triangle^{q}\left(\varphi_{1}, \psi_{1}\right)+\tilde{f}^{q}\left(\varphi_{2}, \psi_{2}\right)\right) \\
& =\left(\delta^{q} \varphi_{2},-\tilde{i}^{q} \varphi_{2}-\delta^{q-1} \psi_{2},-\delta^{q-1} \varphi_{1}+\tilde{f}^{q} \varphi_{2}, \tilde{i}^{q-1} \varphi_{1}+\delta^{q-2} \psi_{1}+\widetilde{f \mid A}\right)^{q-1} \psi_{2} .
\end{aligned}
$$

Then there is a short exact sequence

$$
0 \longrightarrow \stackrel{+}{M}=(X, A) \stackrel{\lambda^{ \pm}}{\longrightarrow} M_{K}^{ \pm}(f) \stackrel{x^{ \pm}}{\longrightarrow} \bar{M}_{K}^{ \pm}(Y, B) \longrightarrow \underline{O}_{2},
$$

where $\lambda^{\neq}, \chi^{\neq}$are injection, projection, respectively; $\stackrel{+}{M}^{\neq}(X, A)$ is the complex $M_{K}^{\neq}(X, A)$ with the dimensions all raised by one, and $\bar{M}^{\neq}(Y, B)$ is the complex $M^{\neq}(Y, B)$ with the 
sign of the coboundary changed [12]. Note that $H^{q}\left(\bar{M}_{K}^{ \pm}(Y, B)\right)=\tilde{H}_{K}^{q}(Y, B)$. Let $V$ be an open subset of $X$ such that $\bar{V} \subseteq \operatorname{Int} A, B=X-V$, and $C=A-V$. Put the excision map $e:(B, C) \hookrightarrow(X, A)$ in (4.3) instead of $f$, and then apply the cohomology functor to get the long exact sequence

$$
\cdots \longrightarrow \tilde{H}_{K}^{q}(e) \stackrel{\tilde{x}^{*}}{\longrightarrow} \tilde{H}_{K}^{q}(X, A) \stackrel{\tilde{e}^{*}}{\longrightarrow} \tilde{H}_{K}^{q}(B, C) \stackrel{\tilde{\lambda}^{*}}{\longrightarrow} \tilde{H}_{K}^{q+1}(e) \longrightarrow \cdots .
$$

Thus the groups $\tilde{H}_{K}^{q}(e), \tilde{H}_{K}^{q+1}(e)$ measure how much the cohomological groups deviate from the excision axiom.

THEOREM 4.1. If $\operatorname{dim} K=0, e:(B, C) \rightarrow(X, A)$ is an excision map, where $A$ is closed and $\left(G, G^{\prime}\right)$ any pair of topological abelian groups, then $\tilde{H}_{K}^{q}(e)=0$ when $q=0$ or $q=1$.

Proof. (1) Case $q=0$. We have

$$
M_{K}^{0}(e)=M_{K}^{0}(X, A)=M_{K}^{0}(X)=L_{K}^{0}(X) .
$$

Let $\varphi \in M_{K}^{0}(e)$ such that $\tilde{\triangle}_{\varphi}=0$, then $\tilde{i}^{0} \varphi=0$, ẽ $\varphi=0$. Then $\varphi=0$ [7], which means that $\operatorname{Ker} \tilde{\triangle}^{0}=0$.

(2) Case $q=1$. We have

$$
M_{K}^{1}(e)=M_{K}^{\prime}(X) \oplus L^{0}(A) \oplus L^{0}(B) .
$$

It is sufficient to show that $\operatorname{Ker} \tilde{\triangle}^{1} \subseteq \operatorname{Im} \tilde{\triangle}^{0}$. Let $\left(\varphi_{2}, \psi_{2}, \varphi_{1}, 0\right) \in \operatorname{Ker} \tilde{\triangle}^{1}$, then

$$
\begin{gathered}
\delta^{1} \varphi=0, \quad \tilde{i}^{\prime} \varphi_{2}=-\delta^{0} \psi_{2}, \\
\tilde{e}^{1} \varphi_{2}=\delta^{0} \varphi_{1}, \\
\tilde{e}_{1}^{0}\left(-\psi_{2}\right)=\tilde{j} \varphi_{1},
\end{gathered}
$$

where $i: A \hookrightarrow X, j: C \hookrightarrow B$ and $e_{1}=e \mid C$.

By (4.9), there exists $\varphi \in M_{K}^{0}(X)$ [7] such that

$$
\tilde{i}^{0} \varphi=-\psi_{2}, \quad \tilde{e}^{0} \varphi=\varphi_{1} .
$$

By (4.8), (4.9), and (4.10), we get

$$
\tilde{i}^{1}\left(\delta^{0} \varphi-\varphi_{2}\right)=0, \quad \tilde{e}^{1}\left(\delta^{0} \varphi-\varphi_{2}\right)=0 .
$$

Then $\delta^{0} \varphi=\varphi_{2}$ [7], which together with (4.11) yield $(\varphi, 0,0,0) \in M_{K}^{0}(e)$ such that $\tilde{\triangle}^{0}(\varphi, 0,0,0)=\left(\varphi_{2}, \psi_{2}, \varphi_{1}, 0\right)$.

Combining the sequence (4.4) and the above theorem, we get the following result.

COROLLARY 4.2. Under the assumptions of Theorem 4.1, the map $\tilde{e}^{* 0}: \tilde{H}_{K}^{0}(X, A) \rightarrow$ $\tilde{H}_{K}^{0}(B, C)$ is an isomorphism but $\tilde{e}^{* 1}$ is a monomorphism.

Next we give a second application to the work introduced in this paper.

Let $\eta:\left(G, G^{\prime}\right) \rightarrow\left(F, F^{\prime}\right)$ be a homeomorphism of pairs of (discrete) abelian groups, which is an epimorphism, $\left(L, L^{\prime}\right)=\operatorname{Ker} \eta$ and $\lambda:\left(L, L^{\prime}\right) \hookrightarrow\left(G, G^{\prime}\right)$. Then for each $\bar{\alpha} \in$ $\Omega(X, A)$, the maps $\eta, \lambda$ define naturally a short exact sequence

$$
0 \rightarrow C^{q}\left(\bar{\alpha}, L, L^{\prime}\right) \rightarrow C^{q}\left(\bar{\alpha} ; G, G^{\prime}\right) \rightarrow C^{q}\left(\bar{\alpha} ; F, F^{\prime}\right) \longrightarrow 0 ;
$$


its cohomology is a long exact sequence [12] denoted by $S_{\bar{\alpha}}$. One can show that $\left\{S_{\bar{\alpha}}\right\}_{\Omega(X, A)}$ is a direct system, its direct limit $[7,8]$ is

$$
\begin{aligned}
\cdots & \rightarrow \bar{H}_{K}^{q-1}\left(X, A ; F, F^{\prime}\right) \longrightarrow \bar{H}_{K}^{q}\left(X, A^{\prime} ; L, L^{\prime}\right) \longrightarrow \bar{H}_{K}^{q}\left(X, A ; G, G^{\prime}\right) \\
& \longrightarrow \bar{H}_{K}^{q}\left(X, A, F, F^{\prime}\right) \longrightarrow \bar{H}_{K}^{q+1}\left(X, A ; L, L^{\prime}\right) \longrightarrow \cdots
\end{aligned}
$$

Now instead of $F$ take the factor group $G / G^{\prime}$ and so $F^{\prime}$ will be the null subgroup of $G / G^{\prime}$. Then the above sequence yields the following result.

THEOREM 4.3. Consider $(X, A)$ has a trivial $(q-1)$-dimensional space $K$-AlexanderSpanier cohomology group with finite cochains, and a trivial $(q+1)$-dimensional $K$ Alexander-Spanier cohomology with infinite cochains, taken over the coefficient groups $G / G^{\prime}$ and $G^{\prime}$, respectively. Then the group $\bar{H}_{K}^{q}\left(X, A ; G, G^{\prime}\right)$ defined over an arbitrary pair $\left(G, G^{\prime}\right)$ of coefficient groups is the extension of the cohomology group $\bar{H}_{K}^{q}\left(X, A ; G^{\prime}\right)$ with infinite cochains over $G^{\prime}$ by the group $\bar{H}_{K}^{q}\left(X, A, G / G^{\prime}\right)$ with finite cochains over $G / G^{\prime}$.

ACKNOWLEDGEment. The first author would like to thank the Abdus Salam International Centre for Theoretical Physics, Trieste, Italy, for hospitality.

\section{REFERENCES}

[1] N. Berikashvili, On axiomatic theory of spectra and duality laws for arbitrary space, Trudy Tbilisi Mat. Inst. 24 (1957).

[2] E. H. Brown Jr. and R. H. Szczarba, Continuous cohomology and real homotopy type, Trans. Amer. Math. Soc. 311 (1989), no. 1, 57-106. MR 89f:55005. Zbl 671.55006.

[3] _ Continuous cohomology and real homotopy type. II, Astérisque (1990), no. 191, 45-70, International Conference on Homotopy Theory (Marseille, Luminy, 1988). MR 92d:55004. Zbl 731.55008.

[4] G. S. Chogoshvili, Ueber Homologieapproximationen und Dualitaetssaetze fuer beliebige Mengen [On homological approximation and duality laws for arbitrary space], Mat. Sb. (N.S.) 28 (1951), no. 70, 89-118 (Russian). Zbl 042.16903.

[5] A. E. A. Dabbour, Ueber die Baladzeschen Homologiegruppen kompakter Raeume [On The Baladze homology groups of compact spaces], Soobshch. Akad. Nauk Gruzin. SSR 77 (1975), no. 3, 541-544 (Russian). MR 52\#9213. Zbl 323.55012.

[6] On the K-Kolmogorov homology and cohomology construction, Simon Stevin 66 (1992), no. 3-4, 357-387. MR 94k:55013. Zbl 780.55005.

[7] A. E. A. Dabbour and Hijazi R. A., Axiomatic characterization of Alexander-Spanier Ktypes, ICTP preprint IC/96/152, Bull. Pure Appl. Sci. Sec. E Math. 17 (1998).

[8] _ The K-Alexander-Spanier cohomology, ICTP preprint IC/95/231, Bull. Pure Appl. Sci. Sec. E Math. 17 (1998).

[9] S. Eilenberg and N. Steenrod, Foundations of Algebraic Topology, Princeton Mathematical Series No.15, Princeton University Press, Princeton, 1952. MR 14,398b. Zbl 047.41402.

[10] R. Engelking, General Topology, PWN-Polish Scientific Publishers, Warsaw, 1977, translated from the Polish by the author. Monografie Matematyczne, Tom 60. [Mathematical Monographs, vol. 60]. MR 58\#18316b. Zbl 373.54002.

[11] S.-t. Hu, Cohomology theory in topological groups, Michigan Math. J. 1 (1952), 11-59. MR 14,449b. Zbl 048.25802.

[12] S. Mac Lane, Homology, Classics in Mathematics, Springer-Verlag, Berlin, 1995, Reprint of the 1975 edition. MR 96d:18001. Zbl 818.18001. 
[13] W. S. Massey, A Basic Course in Algebraic Topology, Graduate Texts in Mathematics, 127, Springer-Verlag, New York, 1991. MR 92c:55001. Zbl 725.55001.

[14] L. M. Mdzinarishvili, Partially continuous Alexander-Spanier cohomology theory, 130 (1996), 1-39, Mathematical Institute of University Heidelberg.

[15] M. A. Mostow, Continuous cohomology of spaces with two topologies, Mem. Amer. Math. Soc. 7 (1976), no. 175, x+142. MR 54\#1253. Zbl 343.55004.

[16] C. W. Patty, Foundations of Topology, The Prindle, Weber and Schmidt Series in Advanced Mathematics, PWS-KENT Publishing Co., Boston, 1993. MR 94d:54002. Zbl 931.54001.

[17] E. H. Spanier, Cohomology theory for general spaces, Ann. of Math. (2) 49 (1948), 407-427. MR 9,523d. Zbl 035.24801.

[18] _ Algebraic Topology, McGraw-Hill Series in Higher Mathematics, McGraw-Hill Book, New York, 1966. MR 35\#1007. Zbl 145.43303.

Abd El-Sattar A. Dabbour: Department of Mathematics, Faculty of Science, AIN-SHAMS UNIVERSITY, ABBASIA, CAIRo, EgYPT

Current address: The Abdus SAlam InTernational Centre for Theoretical Physics, TRIESTE, ITALY

E-mail address: sherif@sunet.shams.eg

Rola A. Hijazi: Department of Mathematics, Faculty of SCIENCE, King AbDUlaziz UniVERSITY, P.O. BOX 14466, JEDDAH 21424, SAUDI ARABIA 


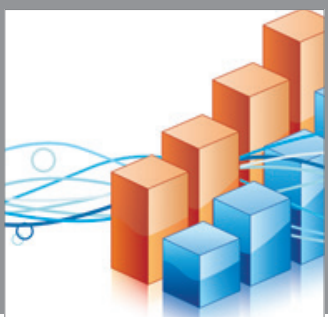

Advances in

Operations Research

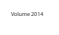

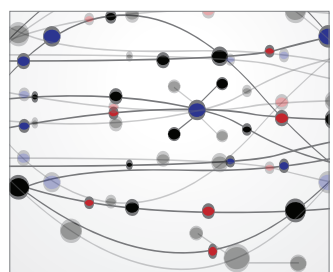

\section{The Scientific} World Journal
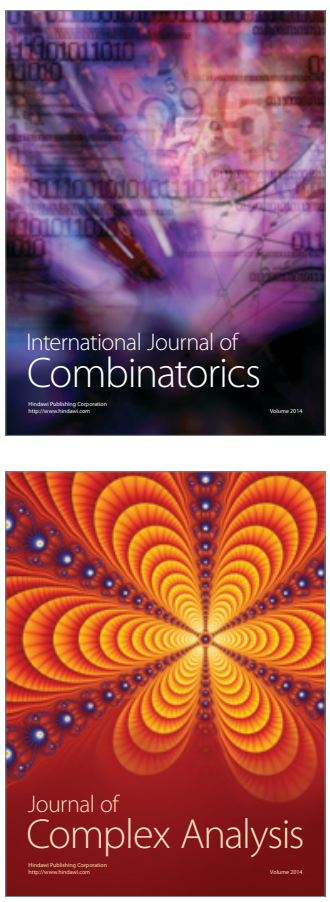

International Journal of

Mathematics and

Mathematical

Sciences
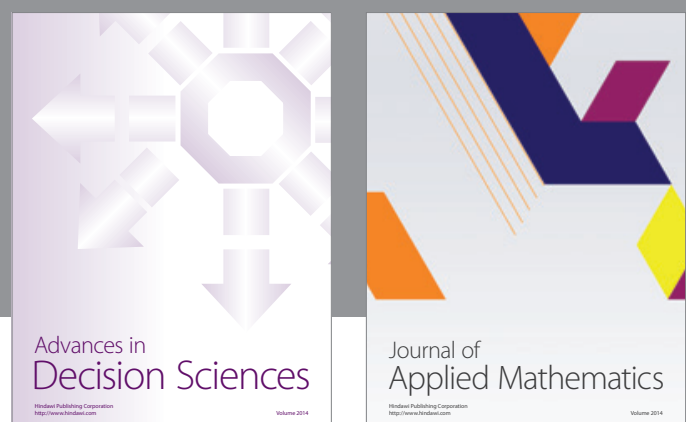

Journal of

Applied Mathematics
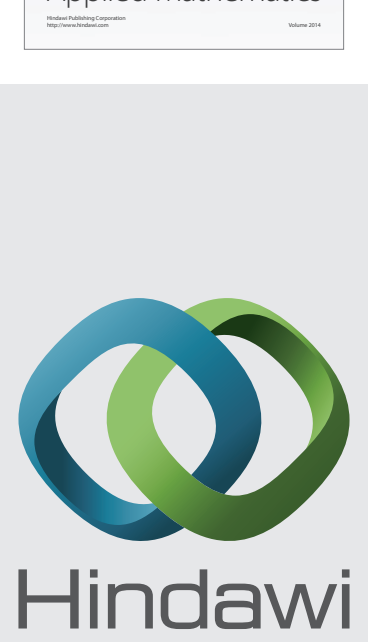

Submit your manuscripts at http://www.hindawi.com
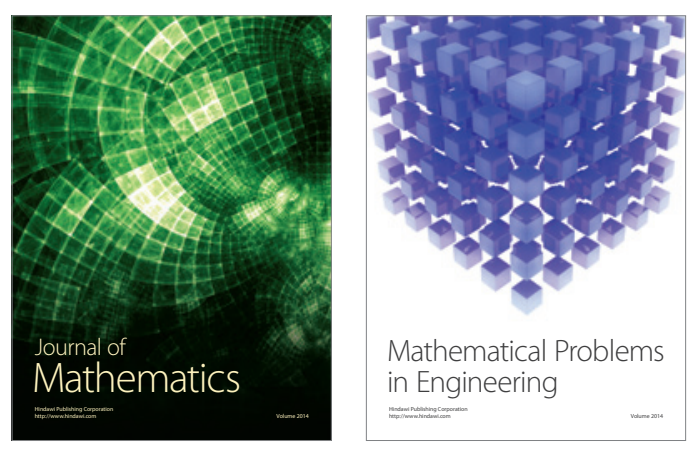

Mathematical Problems in Engineering
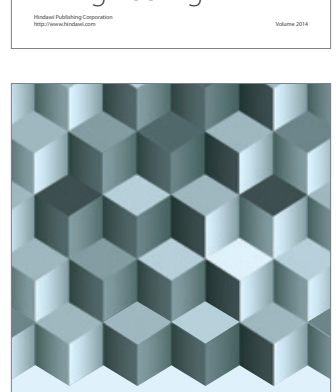

Journal of

Function Spaces
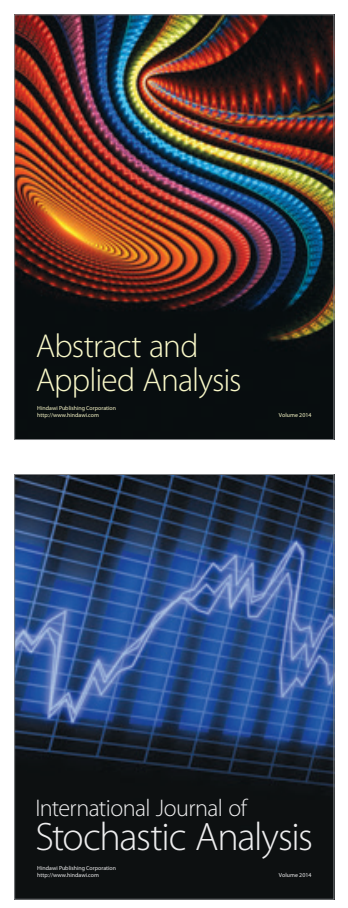

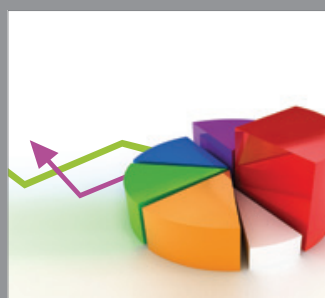

ournal of

Probability and Statistics

Promensencen
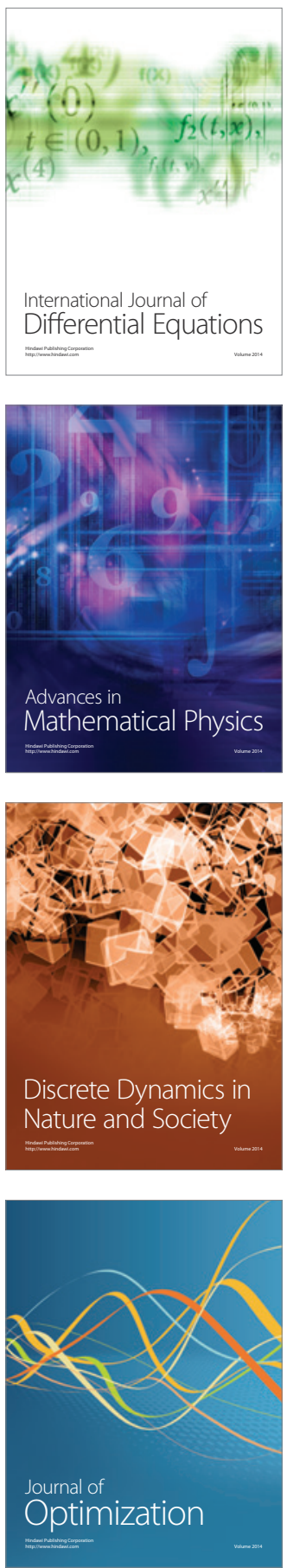